University of Nebraska - Lincoln

DigitalCommons@University of Nebraska - Lincoln

$11-5-2020$

\title{
Detection of uncompensated magnetization at the interface of an epitaxial antiferromagnetic insulator
}

\author{
Pavel N. Lapa \\ University of California, San Diego \\ Min Han Lee \\ University of California, San Diego \\ Igor V. Roshchin \\ Texas A\&M University \\ Kirill Belashchenko \\ University of Nebraska - Lincoln, belashchenko@unl.edu \\ Ivan K. Schuller \\ University of California, San Diego
}

Follow this and additional works at: https://digitalcommons.unl.edu/physicsbelashchenko

Part of the Atomic, Molecular and Optical Physics Commons, Condensed Matter Physics Commons, Engineering Physics Commons, Other Materials Science and Engineering Commons, and the Statistical, Nonlinear, and Soft Matter Physics Commons

Lapa, Pavel N.; Lee, Min Han; Roshchin, Igor V.; Belashchenko, Kirill; and Schuller, Ivan K., "Detection of uncompensated magnetization at the interface of an epitaxial antiferromagnetic insulator" (2020). Kirill Belashchenko Publications. 39.

https://digitalcommons.unl.edu/physicsbelashchenko/39

This Article is brought to you for free and open access by the Research Papers in Physics and Astronomy at DigitalCommons@University of Nebraska - Lincoln. It has been accepted for inclusion in Kirill Belashchenko Publications by an authorized administrator of DigitalCommons@University of Nebraska - Lincoln. 


\title{
Detection of uncompensated magnetization at the interface of an epitaxial antiferromagnetic insulator
}

\author{
Pavel N. Lapa, ${ }^{1}$ Min-Han Lee $\odot,{ }^{1,2}$ Igor V. Roshchin, ${ }^{3}$ Kirill D. Belashchenko $\odot,{ }^{4}$ and Ivan K. Schuller ${ }^{1}$ \\ ${ }^{1}$ Department of Physics, University of California, San Diego, La Jolla, California 92093, USA \\ ${ }^{2}$ Materials Science and Engineering Program, University of California San Diego, La Jolla, California 92093, USA \\ ${ }^{3}$ Department of Material Science and Engineering, Texas A\&M University, College Station, Texas 77843-4242, USA \\ ${ }^{4}$ Department of Physics and Astronomy and Nebraska Center of Materials and Nanoscience, University of Nebraska-Lincoln, \\ Lincoln, Nebraska 68588-0299, USA
}

(Received 25 May 2020; revised 21 October 2020; accepted 23 October 2020; published 5 November 2020; corrected 20 November 2020)

\begin{abstract}
We have probed directly the temperature and magnetic field dependence of pinned uncompensated magnetization at the interface of antiferromagnetic $\mathrm{FeF}_{2}$ with $\mathrm{Cu}$, using $\mathrm{FeF}_{2}-\mathrm{Cu}-\mathrm{Co}$ spin valves. Electrons polarized by the Co layer are scattered by the pinned uncompensated moments at the $\mathrm{FeF}_{2}-\mathrm{Cu}$ interface giving rise to giant magnetoresistance. We determined the direction and magnitude of the pinned uncompensated magnetization at different magnetic fields and temperatures using the angular dependencies of resistance. The strong $\mathrm{FeF}_{2}$ anisotropy pins the uncompensated magnetization along the easy axis independent of the cooling field orientation. Most interestingly, magnetic fields as high as $90 \mathrm{kOe}$ cannot break the pinning at the $\mathrm{FeF}_{2}-\mathrm{Cu}$ interface. This proves that the pinned interfacial magnetization is strongly coupled to the antiferromagnetic order inside the bulk $\mathrm{FeF}_{2}$ layer. Studies as a function of $\mathrm{FeF}_{2}$ crystalline orientation show that uncompensated spins are only detected in a spin valve with (110) crystal orientation, but not in valves containing $\mathrm{FeF}_{2}(100)$ and $\mathrm{FeF}_{2}(001)$. This observation is in agreement with symmetry-related considerations which predict the equilibrium boundary magnetization for the $\mathrm{FeF}_{2}$ (110) layer.
\end{abstract}

DOI: 10.1103/PhysRevB.102.174406

\section{INTRODUCTION}

Antiferromagnets are essential for the development of spintronics applications [1]. They display faster dynamics than ferromagnets, which can be exploited in devices operating at terahertz frequencies [2,3]. Vanishing macroscopic magnetization eliminates stray fields, making antiferromagnetic devices insensitive to external magnetic fields. The major drawback is that the antiferromagnetic order cannot be easily manipulated nor detected using commonly available techniques. Moreover, laboratory magnetic fields can only affect uncompensated magnetic moments present due to defects.

However, local compensation of magnetic moments in bulk antiferromagnets may not be preserved at an interface either for symmetry reasons or because of imperfections. Moreover, the magnetic-moment magnitude and orientation at interfaces may vary significantly depending on the crystalline orientation of the antiferromagnetic layer [4]. For certain orientations of an antiferromagnetic layer, the interfacial spin structure may be uncompensated, enabling useful functionality in magnetic heterostructures. For example, pinning of the uncompensated interfacial magnetization is responsible for exchange bias in ferromagnet/antiferromagnet heterostructures [4-10]. Additionally, giant and tunneling magnetoresistance, extensively studied in ferromagnetic spin valves, may exhibit different properties when ferromagnets are substituted by antiferromagnets [11,12]. Low spin accumulation at interfaces with antiferromagnets may be a hindrance for the development of antiferromagnetic spin valves for practical applications. However, they may provide crucial information on the interfacial uncompensated spin configuration. This information is necessary for further development of antiferromagnetic spintronics and is difficult to obtain by other experimental techniques.

Crucial insight into the surface magnetism of antiferromagnets can be inferred from symmetry considerations. A single-domain antiferromagnet, without time-reversal symmetry in its magnetic point group, has an uncompensated magnetization at a generically oriented surface, which is thermodynamically stable and protected against surface roughness $[13,14]$. The lack of macroscopic time-reversal symmetry in an antiferromagnet is associated with linear or nonlinear magnetoelectricity [15]. In particular, it was demonstrated that the boundary magnetization of a linear magnetoelectric can be switched by a combination of external electric and magnetic fields $[14,16]$. The linear magnetoelectric effect is forbidden by inversion symmetry in transition-metal fluorides with the rutile lattice, such as $\mathrm{FeF}_{2}$, but a time-reversal-breaking single-domain state can still be achieved by direct coupling of the boundary magnetization to an external magnetic field.

In this paper, we use antiferromagnet-ferromagnet spin valves (AFSVs) [11] to study the uncompensated magnetization and its pinning at the surface of differently oriented antiferromagnetic $\mathrm{FeF}_{2}$ layers. The valves consist of an epitaxially grown $\mathrm{FeF}_{2}$ layer and a ferromagnetic Co layer separated by a thin $\mathrm{Cu}$ spacer. The purpose of these spin valves is to detect giant magnetoresistance (GMR) which depends on 


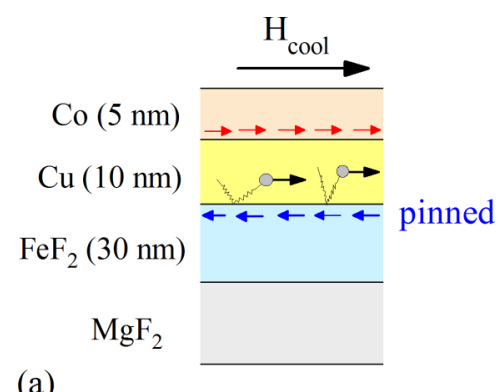

(a)

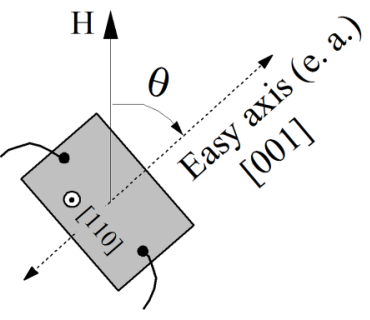

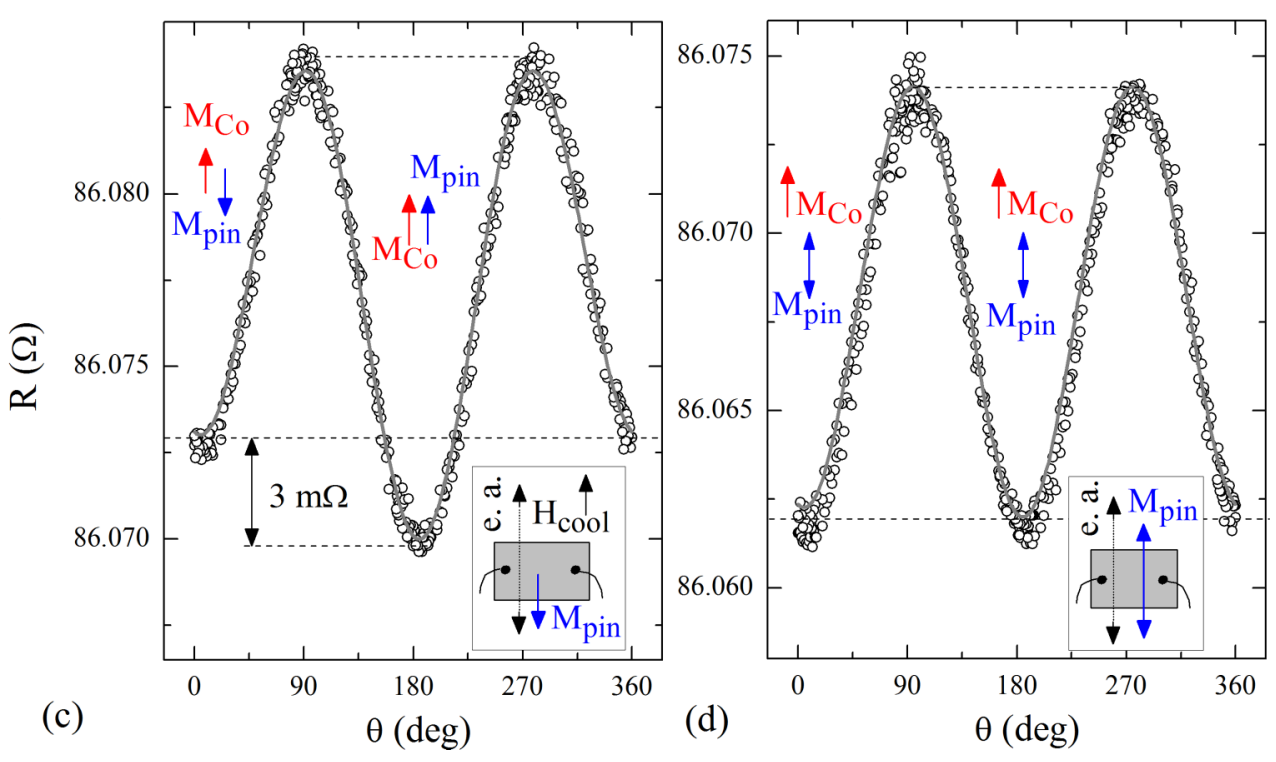

FIG. 1. (a) Schematics illustrates scattering of electrons polarized by the Co layer on the pinned uncompensated magnetization at the $\mathrm{FeF}_{2}-\mathrm{Cu}$ interface. (b) Schematics shows rotation of the $\mathrm{FeF}_{2}(110) / \mathrm{Cu} / \mathrm{Co}$ valve in an external magnetic field; easy axis (e.a.) is perpendicular to the long edge of the sample; the sample orientation is defined by an angle $\theta$ between the direction of magnetic field and the easy axis. Angular dependencies of resistance $R$ for the $\mathrm{FeF}_{2}(110) / \mathrm{Cu} / \mathrm{Co}$ AFSV measured at $10 \mathrm{~K}$ in a $90-\mathrm{kOe}$ magnetic field after cooling the valve from $300 \mathrm{~K}$ (c) in the $90-\mathrm{kOe}$ magnetic field applied along the easy axis of $\mathrm{FeF}_{2}\left(\theta_{\text {cool }}=0^{\circ}\right)$, (d) after cooling in zero magnetic field (ZFC). Circles show experimental data, grey lines are the fits to Eq. (1). Blue and red arrows show the orientation of the pinned uncompensated magnetization $\left(M_{\text {pin }}\right)$ at the $\mathrm{FeF}_{2}-\mathrm{Cu}$ interface (assuming the normal $\left.\mathrm{GMR}\right)$ and the Co layer $\left(M_{C o}\right)$, respectively. The schematics in the boxes at (c) and (d) illustrate the mutual orientation of cooling magnetic field $\left(H_{\text {cool }}\right), M_{\text {pin }}$, and the FeF $F_{2}$ easy axis for the particular field-cooling procedures.

the mutual orientation of the magnetizations in the Co layer and at the $\mathrm{FeF}_{2}-\mathrm{Cu}$ interface [Fig. 1(a)]. In this context, the most useful aspect of $\mathrm{FeF}_{2}$ is that it is an insulating antiferromagnet, therefore only the $\mathrm{FeF}_{2}-\mathrm{Cu}$ interface is accessible to free charge carriers in an AFSV. Because of this, certain features of the magnetoresistance may be directly attributed to the uncompensated magnetization at this interface.

Antiferromagnetic insulators are of interest due to their possible applications in low-power magnonic devices [17,18]. $\mathrm{FeF}_{2}$ is a well-studied antiferromagnet with a tetragonal rutile structure and simple Néel spin ordering. Below the Néel temperature $(78 \mathrm{~K})$, the strong magnetic anisotropy provides high stability of this spin structure along the easy, $c$ axis ([001] direction). As discussed below, symmetry considerations show that the (110) surface of $\mathrm{FeF}_{2}$ has a thermodynamically stable macroscopic uncompensated magnetization, whereas (001) and (100) surfaces do not. This feature is consistent with the fact that the magnitude of the exchange bias in $\mathrm{FeF}_{2}$ /ferromagnet systems strongly depends on the $\mathrm{FeF}_{2}$ crystallographic orientation [4]. Although the interfacial magnetization is much lower than that in the Co layer, the AFSV technique enabled us to detect and study its behavior in a wide range of temperatures and magnetic fields. Specifically, the measurements reveal some peculiar properties of the interfacial magnetization which are distinctively different from its ferromagnetic counterpart: the interfacial magnetization exhibits unprecedented stability, and magnetization reversal can only be observed at temperatures close to the Néel temperature.

\section{EXPERIMENTAL DETAILS}

$\mathrm{FeF}_{2}(30 \mathrm{~nm}) / \mathrm{Cu}(10 \mathrm{~nm}) / \mathrm{Co}(5 \mathrm{~nm}) / \mathrm{Ti}(5 \mathrm{~nm})$ AFSVs were grown using electron-beam gun evaporation on $\mathrm{MgF}_{2}$ (110), $\mathrm{MgF}_{2}$ (100), and $\mathrm{MgF}_{2}$ (001) single-crystal substrates. The $\mathrm{FeF}_{2}$ layer was grown at $300{ }^{\circ} \mathrm{C}$ at a rate of $0.3 \AA / \mathrm{sec}$. To avoid interdiffusion, the rest of the layers $(\mathrm{Cu}, \mathrm{Co}, \mathrm{Ti})$ were grown at a rate of $0.5 \AA / \mathrm{sec}$ after the substrates were cooled down to $50^{\circ} \mathrm{C} . \mathrm{MgF}_{2}$ and $\mathrm{FeF}_{2}$ have closely matching crystallographic structures and lattice parameters which allows for epitaxial growth of $\mathrm{FeF}_{2}(110), \mathrm{FeF}_{2}(100)$, and $\mathrm{FeF}_{2}(001)$ on the $\mathrm{MgF}_{2}$ substrates. The corresponding AFSVs are denoted as $\mathrm{FeF}_{2}(110) / \mathrm{Cu} / \mathrm{Co}, \mathrm{FeF}_{2}(100) / \mathrm{Cu} / \mathrm{Co}$, and $\mathrm{FeF}_{2}(001) / \mathrm{Cu} / \mathrm{Co}$ further in the text.

Four-probe electrical resistivity measurements were performed on $8 \mathrm{~mm} \times 2 \mathrm{~mm}$ stripes with the current injected along the long edge. For the $\mathrm{FeF}_{2}(110) / \mathrm{Cu} / \mathrm{Co}$ and $\mathrm{FeF}_{2}(100) / \mathrm{Cu} / \mathrm{Co}$ AFSVs, the $\mathrm{FeF}_{2}$ [001] easy axis is perpendicular to the long edge of the stripes. The spin valves were installed on a horizontal rotator, i.e., with the external magnetic field in the plane of the film. The angular orientation of AFSVs is defined by the angle $\theta$ between the $\mathrm{FeF}_{2}$ [001] easy axis and the positive magnetic-field direction [Fig. 1(b)]. Thus, $0^{\circ}$ corresponds to the orientation in which the magnetic field is perpendicular to the long edge of the stripes and parallel to the $\mathrm{FeF}_{2}$ easy axis [Fig. 1(b)]. Whereas the results presented in this paper are based on one set of AFSVs, two additional sets of AFSVs confirmed the reproducibility of these results. 


\section{EXPERIMENTAL RESULTS}

To get an insight into pinning processes emerging at the $\mathrm{FeF}_{2}-\mathrm{Cu}$ interface, the following measurement protocol was used. An AFSV was cooled from $300 \mathrm{~K}$ to a temperature $T$ in a magnetic field $H_{\text {cool }}$ while the valve was oriented at $\theta_{\text {cool }}$. The resistance $R$ was then measured as a function of the sample orientation in magnetic field $H_{\text {meas }}$ while $\theta$ was varied from $0^{\circ}$ to $360^{\circ}$ and back to $0^{\circ}$. The angular dependencies of the resistance for a $\mathrm{FeF}_{2}(110) / \mathrm{Cu} / \mathrm{Co}$ AFSV measured after cooling in a $90-\mathrm{kOe}$ magnetic field applied along the easy axis $\left(\theta_{\text {cool }}=0^{\circ}\right)$ and after cooling in zero magnetic field (ZFC) are shown in Figs. 1(c) and 1(d), respectively. The measurements were conducted at $10 \mathrm{~K}$ in the $H_{\text {meas }}=90 \mathrm{kOe}$ magnetic field. Both curves exhibit a harmonic behavior as expected due to anisotropic magnetoresistance (AMR). However, there is a salient feature that cannot be explained by AMR. The curve for the field-cooled AFSV [Fig. 1(c)] is not symmetric under reversal of the magnetic field along the cooling direction: resistance at $\theta=0^{\circ}$ is about $3 \mathrm{~m} \Omega$ higher than at $180^{\circ}$, whereas it is the same at $\theta=90^{\circ}$ and $270^{\circ}$. On the other hand, the resistance of the ZFC sample is symmetric under field reversal [Fig. 1(d)]. The overall vertical shift of the curves in Figs. 1(c) and 1(d) occurs because a lock-in detector got reconfigured between the corresponding measurements. If the cooling magnetic field is reversed $\left(\theta_{\text {cool }}=180^{\circ}\right)$, the global minimum of the resistance is observed at $0^{\circ}$ instead of $180^{\circ}$, and the resistance at $\theta=180^{\circ}$ is $3 \mathrm{~m} \Omega$ higher. These observations clearly indicate that the cooling field yields a preferred orientation that enhances carrier scattering.

Overall, the angular dependence of the resistance can be described as

$$
R(\theta)=R_{0}+\Delta R_{A M R} \sin ^{2}(\theta)+\Delta R_{G M R} \sin ^{2}\left[\left(\theta-\theta_{\text {pin }}\right) / 2\right],
$$

where $R_{0}$ is the resistance of the valve in zero magnetic field, and the second term describes the contribution from AMR. The third term describes a GMR contribution caused by spin-dependent scattering on a magnetization pinned at $\theta_{\text {pin }}$ after field cooling. The fit of the experimental data to Eq. (1) with three adjustable parameters $\Delta R_{A M R}, \Delta R_{G M R}$, and $\theta_{\text {pin }}$ is shown by thin grey lines in Figs. 1(c) and 1(d). For cooling along the easy axis $\left(\theta_{\text {cool }}=0^{\circ}\right.$ and $\left.180^{\circ}\right)$, fitting yields $\theta_{\text {pin }}=\theta_{\text {cool }}+180^{\circ}$.

The temperature dependence of the normalized GMR $\left(\Delta R_{G M R} / R_{0}\right)$ for the $\mathrm{FeF}_{2}(110) / \mathrm{Cu} / \mathrm{Co}$ AFSV is shown in Fig. 2. $\triangle R_{G M R}$ values are obtained by fitting to Eq. (1) angular dependencies of the resistance measured in the $90-\mathrm{kOe}$ magnetic field at a sequence of increasing temperatures. Prior to the measurements, the AFSV was cooled to $10 \mathrm{~K}$ in the 90-kOe magnetic field applied along the easy axis of $\mathrm{FeF}_{2}$. The GMR decreases monotonically with increasing temperature up to $60 \mathrm{~K}$ and then sharply drops to zero.

Figure 3(a) shows the dependencies of $\Delta R_{G M R} / R_{0}$ on $H_{\text {meas }}$ at $10 \mathrm{~K}$ (squares), $65 \mathrm{~K}$ (circles), and $70 \mathrm{~K}$ (stars). For these measurements, the $\mathrm{FeF}_{2}(110) / \mathrm{Cu} / \mathrm{Co}$ valve was first cooled to the "target temperature" in the 90-kOe magnetic field applied along the easy axis $\left(\theta_{\text {cool }}=0^{\circ}\right)$. Then, the magnetic field was decreased to $5 \mathrm{kOe}$, and the angular dependence of the resistance was measured in $H_{\text {meas }}$ which was gradually increased from 5 to $90 \mathrm{kOe}$ and then decreased back to $5 \mathrm{kOe}$.

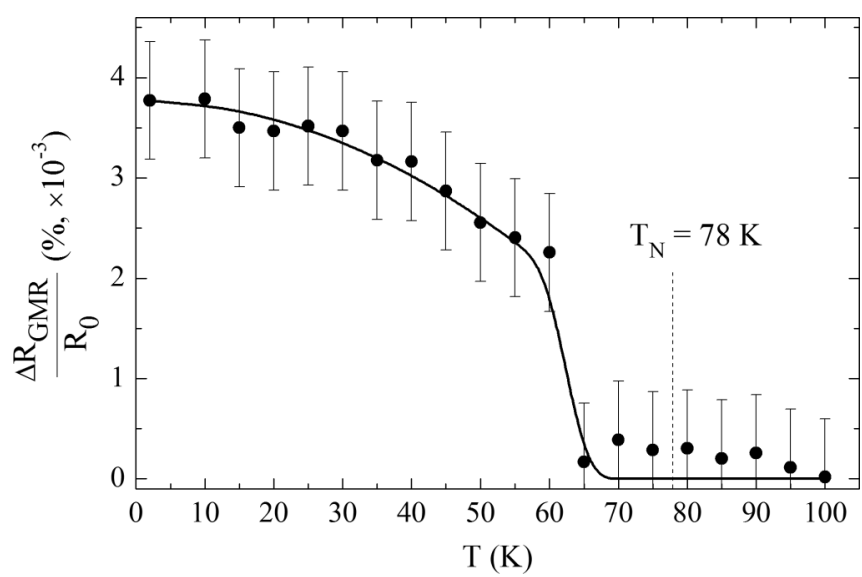

FIG. 2. Temperature dependence of GMR for the $\mathrm{FeF}_{2}(110) / \mathrm{Cu} / \mathrm{Co}$ AFSV measured in a 90-kOe magnetic field after cooling the valve in a $90-\mathrm{kOe}$ magnetic field. The dashed vertical line shows the Néel temperature of bulk $\mathrm{FeF}_{2}$. The black curve is a guide to the eye.

The $H_{\text {meas }}$-up and $H_{\text {meas }}$-down branches of the curves are shown in red and blue colors, respectively. The AFSV was kept at $\theta_{\text {cool }}$ while $H_{\text {meas }}$ was varied. Figure 3(a) shows that the GMR at $10 \mathrm{~K}$ remains constant for all $H_{\text {meas }}$. At the same time, GMR signal measured at 65 and $70 \mathrm{~K}$ becomes almost zero in high magnetic fields. Thus, the GMR signal at $65 \mathrm{~K}$ is significantly suppressed if $H_{\text {meas }}$ is above $80 \mathrm{kOe}$. An increase in temperature by $5 \mathrm{~K}$ causes a significant reduction in the suppression field: the GMR signal at $70 \mathrm{~K}$ disappears if $H_{\text {meas }}$ is above $55 \mathrm{kOe}$.

The angular dependence of the resistance for the $\mathrm{FeF}_{2}(110) / \mathrm{Cu} / \mathrm{Co}$ AFSV was measured at $70 \mathrm{~K}$ in a $10-\mathrm{kOe}$ magnetic field after three different protocols: (1) cooling in a 90-kOe magnetic field at $\theta_{\text {cool }}=0^{\circ}$ and decreasing the field to $10 \mathrm{kOe}$ while the valve is at $0^{\circ}$ [Fig. 3(b)]; (2) after completing the first measurement, rotating the AFSV $180^{\circ}$, applying a 50-kOe magnetic field (which is thus opposite to the cooling field), and measuring at the same 10-kOe magnetic field [Fig. 3(c)]; and (3) after completing the second measurement, applying a larger 70-kOe magnetic field (again opposite to the cooling field) prior to the measurement at $10 \mathrm{kOe}$ [Fig. 3(d)]. Figures $3(\mathrm{~b})$ and $3(\mathrm{c})$ show that the resistance at $180^{\circ}$ is about $1.2 \mathrm{~m} \Omega$ lower compared to that at $0^{\circ}, \theta_{\text {pin }}=\theta_{\text {cool }}+180^{\circ}=$ $180^{\circ}$. At the same time, after applying the 70-kOe magnetic field in the direction which is opposite to the cooling field, the resistance becomes about $1 \mathrm{~m} \Omega$ higher at $180^{\circ}$ compared to that at $0^{\circ}$.

All the data for the $\mathrm{FeF}_{2}(110) / \mathrm{Cu} / \mathrm{Co}$ AFSV presented so far were obtained after cooling the valve in a magnetic field applied along the easy axis of $\mathrm{FeF}_{2}\left(\theta_{\text {cool }}=0^{\circ}\right)$. Figure 4(a) shows the angular dependence of the resistance measured at $10 \mathrm{~K}$ in a $90-\mathrm{kOe}$ magnetic field after cooling the same AFSV in a 90-kOe field applied perpendicular to the easy axis $\left(\theta_{\text {cool }}=90^{\circ}\right)$. The angular dependence is similar to the one at $\theta_{\text {cool }}=0^{\circ}$ [Fig. 1(c)]: in both curves, the resistance at $\theta=180^{\circ}$ is lower than at $0^{\circ}$, whereas those at $90^{\circ}$ and $270^{\circ}$ are the same. The only difference between the curves obtained at $\theta_{\text {cool }}=0^{\circ}$ [Fig. 1(c)] and $\theta_{\text {cool }}=90^{\circ}$ [Fig. 4(a)] is 

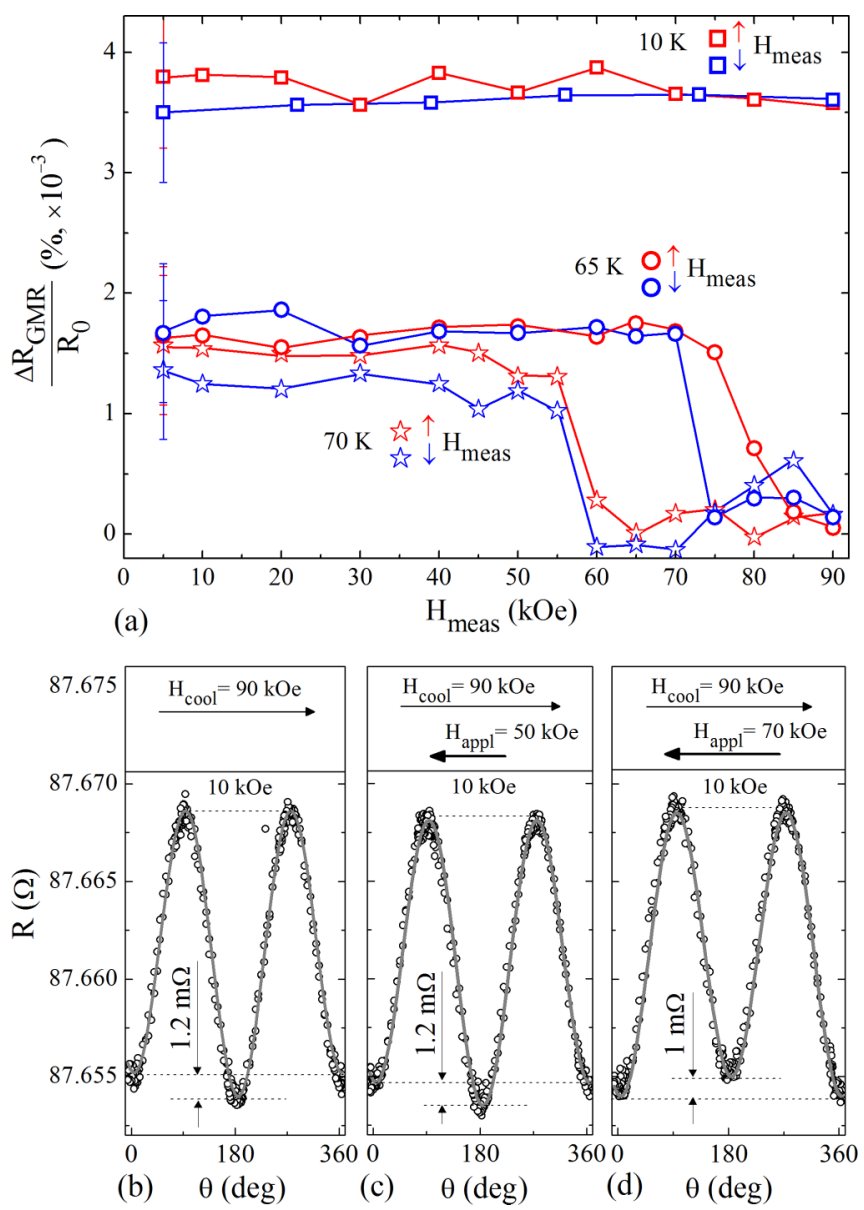

FIG. 3. (a) Magnetic-field dependencies of GMR measured at $10 \mathrm{~K}$ (rectangles), $65 \mathrm{~K}$ (circles), and $70 \mathrm{~K}$ (stars). The data were obtained by cooling the valve to the corresponding temperature in a 90-kOe magnetic field and sequentially measuring the angular dependence incrementally ramping up $H_{\text {meas }}$ (red curves) and then down (blue curves). The error bars in (a) are shown only for a 5-kOe data point. (b) Angular dependencies of resistance measured at $70 \mathrm{~K}$ in a 10-kOe magnetic field after cooling the $\mathrm{FeF}_{2}(110) / \mathrm{Cu} / \mathrm{Co}$ AFSV in the 90-kOe magnetic field applied along the easy axis of $\mathrm{FeF}_{2}\left(\theta_{\text {cool }}=0^{\circ}\right)$; the same dependencies measured in the $10-\mathrm{kOe}$ magnetic field after applying (c) 50-kOe and (d) 70-kOe magnetic field $\left(H_{\text {appl }}\right)$ in the direction opposite to the cooling field $\left(H_{\text {cool }}\right)$. In (b)-(d), circles are experimental data, grey lines are the fits to Eq. (1).

that the GMR $\left(\triangle R_{G M R}\right)$ is slightly lower for the latter curve ( 3 vs $2.6 \mathrm{~m} \Omega$ ). For $\theta_{\text {cool }}=270^{\circ}$ (not shown), the resistance at $180^{\circ}$ becomes higher than the resistance at $0^{\circ}$, and the resistances at $90^{\circ}$ and $270^{\circ}$ remain the same. The fits of the data to Eq. (1) yield $\theta_{\text {pin }}$ is $180^{\circ}$ and $0^{\circ}$ for $\theta_{\text {cool }}=90^{\circ}$ and $\theta_{\text {cool }}=270^{\circ}$, respectively.

Further measurements reveal that as $\theta_{\text {cool }}$ is increased slightly above $90^{\circ}, \theta_{\text {pin }}$ experiences an abrupt change by $180^{\circ}$. This is inferred from the angular dependencies of the resistance measured after cooling the valve in $90 \mathrm{kOe}$ at $\theta_{\text {cool }}=70^{\circ}$ [Fig. 4(b)] and $\theta_{\text {cool }}=110^{\circ}$ [Fig. 4(c)]. The resistance asymmetry along the easy axis implies that $\theta_{\text {pin }}=180^{\circ}$ after the $70^{\circ}$ cooling and $\theta_{\text {pin }}=0^{\circ}$ after the $110^{\circ}$ cooling. Thus, $\theta_{\text {pin }}$ switches by $180^{\circ}$ when the cooling angle is $90^{\circ}<$ $\theta_{\text {cool }}<110^{\circ}$.
The same measurement protocols as were used for the $\mathrm{FeF}_{2}(110) / \mathrm{Cu} / \mathrm{Co}$ valve were applied to the $\mathrm{FeF}_{2}(100) / \mathrm{Cu} / \mathrm{Co}$ and $\mathrm{FeF}_{2}(001) / \mathrm{Cu} / \mathrm{Co}$ AFSVs. No GMR signal was detected for these AFSVs. Since the easy [001] axis is perpendicular to the $\mathrm{FeF}_{2}(001) / \mathrm{Cu} / \mathrm{Co}$ valve's plane, this valve was also installed on a vertical rotator, and a number of $R(\theta)$ curves were measured in a 90-kOe magnetic field rotated out of plane. The $90-\mathrm{kOe}$ magnetic field is sufficiently strong to overcome the shape anisotropy of the Co layer, ensuring that the Co layer still behaves like a free layer and its magnetization rotates out of plane in unison with the external magnetic field. No GMR signal was detected for these out-of-plane measurements of the $\mathrm{FeF}_{2}(001) / \mathrm{Cu} / \mathrm{Co}$ AFSV.

\section{DISCUSSION}

\section{A. Origin of GMR in the $\mathrm{FeF}_{2} / \mathrm{Cu} / \mathrm{Co}$ valve}

The central experimental result is that the resistance of the $\mathrm{FeF}_{2}(110) / \mathrm{Cu} / \mathrm{Co}$ AFSV acquires a GMR-like contribution when the valve is field cooled below the Néel temperature of $\mathrm{FeF}_{2}$ [Fig. 1(c)]. We developed a model which explains the origin of this GMR signal and describes the magnetization processes occurring in a $\mathrm{FeF}_{2}$ antiferromagnet at different temperatures and magnetic fields. A crucial assumption of the model is that cooling in a field only influences the insulating $\mathrm{FeF}_{2}$. Therefore, the change in the magnetoresistance for different cooling procedures can only be related to the magnetization processes at the $\mathrm{FeF}_{2}-\mathrm{Cu}$ interface. The model assumes that the uncompensated magnetization at the $\mathrm{FeF}_{2}-\mathrm{Cu}$ interface becomes pinned along a well-defined direction after field cooling, and scattering of the electrons polarized by the Co layer by this pinned magnetization produces the GMR [Fig. 1(a)]. When the valve is rotated, the magnetization in the Co layer [red arrow in Figs. 1(c) and 1(d)] remains aligned along $H_{\text {meas }}$, while the pinned magnetization rotates with the valve [blue arrow in Figs. 1(c) and $1(\mathrm{~d})]$.

The GMR depends on the mutual orientation of the magnetization in the Co layer and the pinned magnetization at the $\mathrm{FeF}_{2}-\mathrm{Cu}$ interface. The GMR reaches its extrema when these magnetizations are collinear (i.e., parallel or antiparallel). The experimental angular dependencies of the resistance are fitted to Eq. (1), which contains the term describing the normal GMR. The normal GMR implies that the valve's resistance reaches maximum and minimum values when the magnetizations in the Co layer and at the $\mathrm{FeF}_{2}-\mathrm{Cu}$ interface are antiparallel and parallel, respectively. However, since $\mathrm{FeF}_{2}$ and $\mathrm{Co}$ are dissimilar materials, an inverse GMR may be realized in the $\mathrm{FeF}_{2} / \mathrm{Cu} / \mathrm{Co}$ valves. In this case, the resistance maximum and minimum occur when the magnetizations are parallel and antiparallel, respectively. Determining the type of the GMR effect realized in the AFSVs requires calculations of a spin-polarized electronic band structure which goes beyond the scope of this work. Therefore, we assume that $\theta_{\text {pin }}$ obtained from a fit to Eq. (1) determines only the axis along which the magnetization is pinned but not its sign (direction along that axis). However, since the sign of the GMR remains the same for different measurements of the same system, the 

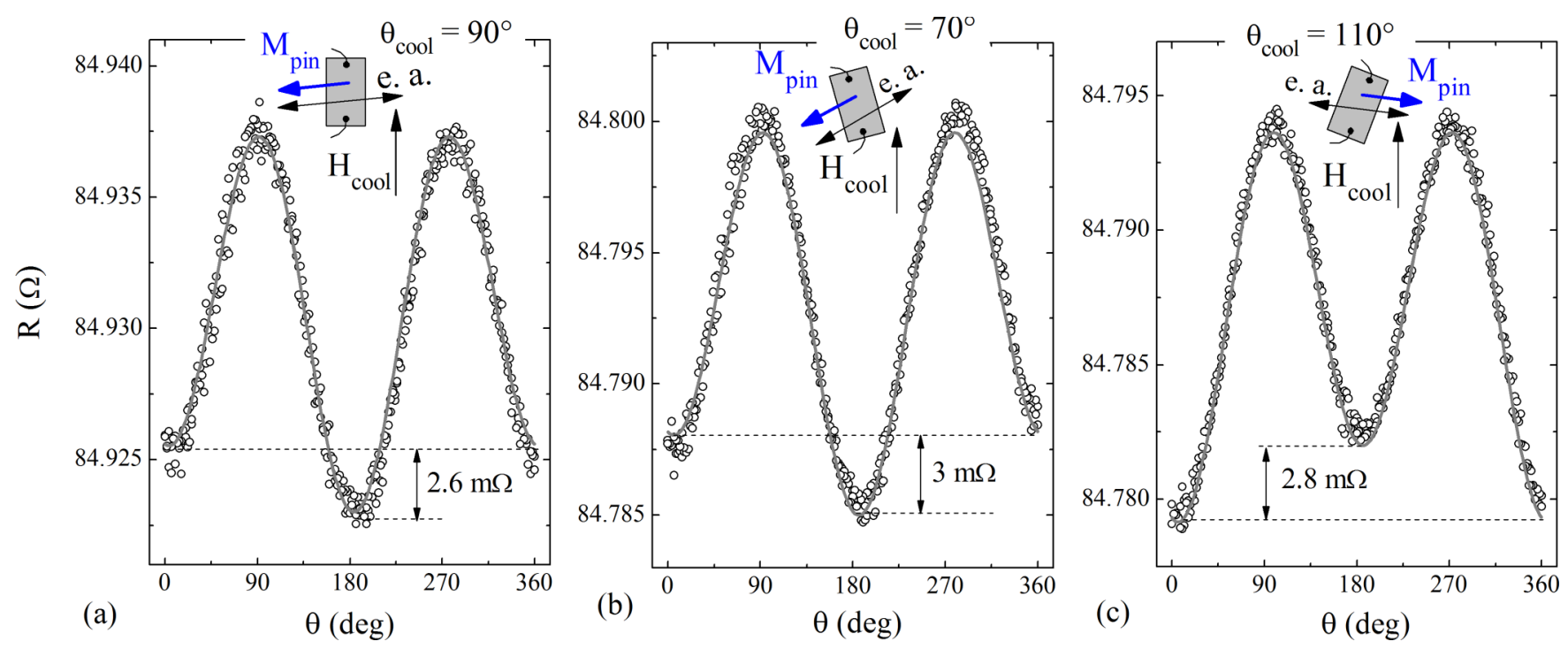

FIG. 4. Angular dependencies of resistance for the $\mathrm{FeF}_{2}(110) / \mathrm{Cu} / \mathrm{Co} \mathrm{AFSV}$ measured at $10 \mathrm{~K}$ in a 90-kOe magnetic field after cooling the valve from $300 \mathrm{~K}$ in the $90-\mathrm{kOe}$ magnetic field while sample orientations are (a) $\theta_{\text {cool }}=90^{\circ}$, (b) $\theta_{\text {cool }}=70^{\circ}$, (c) $\theta_{\text {cool }}=110^{\circ}$. Circles are experimental data, grey lines are the fits to Eq. (1). The schematics at top of each plot illustrate the mutual orientation of the easy axis (e.a.) and the pinned magnetization $\left(M_{\text {pin }}\right)$ (assuming the normal GMR) with respect to the cooling field $\left(H_{\text {cool }}\right)$ direction. Schematic at top corner at (a) explicitly illustrates that the easy axis is not completely perpendicular to the long edge of the sample, consequently, a projection of $H_{\text {cool }}$ on the easy axis defines the direction of the pinned magnetization.

GMR is sensitive to the reversal of the pinned magnetization by $180^{\circ}$.

\section{B. Field cooling and the anisotropy of the boundary magnetization}

If the valve is cooled in a magnetic field along the easy axis $\left(\theta_{\text {cool }}=0^{\circ}\right.$ or $\left.180^{\circ}\right), \theta_{\text {pin }}=\theta_{\text {cool }}+180^{\circ}$ (assuming the normal GMR) or $\theta_{\text {pin }}=\theta_{\text {cool }}$ (assuming inverse GMR). Hence, for cooling along the easy axis, the pinned uncompensated magnetization at the $\mathrm{FeF}_{2}-\mathrm{Cu}$ interface aligns parallel to the cooling field axis. The observation of finite $\Delta R_{G M R}$ even in $H_{\text {meas }}=90 \mathrm{kOe}[$ Fig. 1(c)] indicates that pinning is unusually strong. On the other hand, when the AFSV is cooled in zero field, the GMR signal is zero, which implies that the pinned uncompensated magnetization in that case is randomly distributed in both directions along the $\mathrm{FeF}_{2}$ easy axis [schematics in Fig. 1(d)].

The angular dependence of the resistance measured after cooling the valve in the 90-kOe magnetic field applied perpendicular to the easy axis [Fig. 4(a)] demonstrates another unusual aspect of this pinning. Although the valve was cooled in a very strong magnetic field (90 kOe), the interfacial $\mathrm{FeF}_{2}-\mathrm{Cu}$ magnetization is pinned perpendicular to the cooling field and parallel to the easy axis. The reason for this is that the magnetic field cannot be aligned perfectly perpendicular to the easy axis, i.e., it always has a finite projection along this axis, and the pinned magnetization aligns in the direction of this projection. Since $\theta_{\text {pin }}=180^{\circ}$ (assuming normal GMR) for the nominal $\theta_{\text {cool }}=90^{\circ}$ [Fig. 4(a)], the easy axis is slightly rotated anticlockwise with respect to the long edge of the AFSV, as shown in the schematic in Fig. 4(a). Therefore, if $\theta_{\text {cool }}$ is decreased, $\theta_{\text {pin }}$ should remain at $180^{\circ}$. On the other hand, at a certain orientation where $\theta_{\text {cool }}$ is greater than $90^{\circ}$, the cooling field projection along the easy axis should change sign which would reverse the pinned magnetization, i.e., $\theta_{\text {pin }}$ should switch from $180^{\circ}$ to $0^{\circ}$. These expectations are confirmed by the angular dependencies of the resistance shown in Figs. 4(b) and 4(c), which clearly show that $\theta_{\text {pin }}$ is $180^{\circ}$ and $0^{\circ}$ for $\theta_{\text {cool }}=70^{\circ}$ and $\theta_{\text {cool }}=110^{\circ}$, respectively. Thus, we concluded that, at low temperatures, the magnetization at the $\mathrm{FeF}_{2}(110) / \mathrm{Cu}$ interface is always pinned along the easy axis, and its direction is determined by the projection of the cooling field on the easy axis. This is similar to the observations made by Olamit and co-workers $[19,20]$ for an exchange bias bilayer system $\mathrm{FeF}_{2} / \mathrm{Co}$.

The large anisotropy in $\mathrm{FeF}_{2}$ is responsible for the direction in which the surface magnetization is pinned upon field cooling. However, in general, an uncompensated surface magnetization anisotropy could be different from that in the bulk of an antiferromagnet. The fact that the two anisotropies coincide is additional proof of the strong coupling of the surface uncompensated magnetization with the bulk antiferromagnetic order parameter, which, in turn, is an additional indirect confirmation for the proposed mechanism of the origin of the uncompensated magnetization at the [110] surface of $\mathrm{FeF}_{2}$.

\section{Origin of the macroscopic boundary magnetization in $\mathrm{FeF}_{2}$ from the symmetry analysis}

Symmetry considerations give further insight into the observed behavior. To determine whether a given interface of $\mathrm{FeF}_{2}$ has an equilibrium magnetization, we need to identify the subgroup of its bulk magnetic point group $4^{\prime} / \mathrm{mm}^{\prime} \mathrm{m}$ that leaves the normal vector of the given surface invariant and determine whether this subgroup is compatible with ferromagnetism, i.e., whether it allows an invariant axial vector 


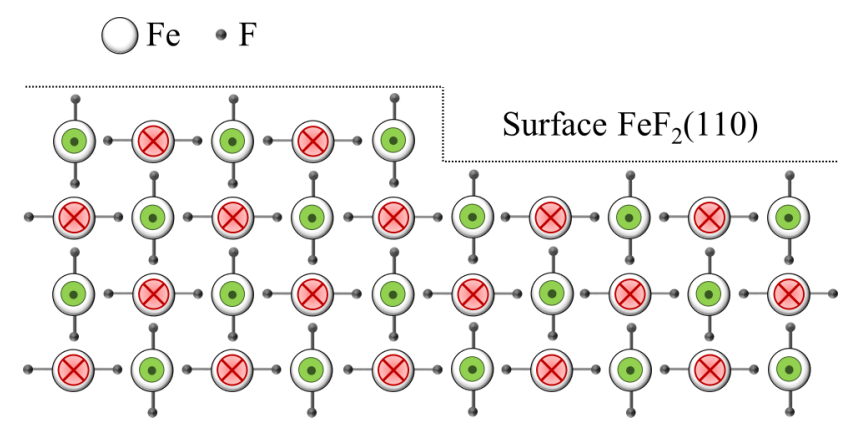

FIG. 5. Schematics illustrates the crystallographic and spin structures of the $\mathrm{FeF}_{2}(110)$ layer with an atomic step. The green and red circles depict the Fe spins pointed in and out of the page, respectively, along the easy axis of $\mathrm{FeF}_{2}$.

[14]. These subgroups are determined by inspection: $4^{\prime} \mathrm{m}^{\prime} \mathrm{m}$ for the (001) surface, $m m 2$ for (100), and $m^{\prime} m 2^{\prime}$ for (110). Of these groups, only $m^{\prime} m 2^{\prime}$ is compatible with ferromagnetism. Thus, on a macroscopic scale, the (110) surface of $\mathrm{FeF}_{2}$ has a net magnetization, whereas the (001) and (100) surfaces are fully compensated. This analysis shows that, for the $\mathrm{FeF}_{2}$ (110) layer, the uncompensated surface magnetization is preserved even if the surface is not atomically flat. In contrast, for the (001) and (100) $\mathrm{FeF}_{2}$ layers, surface roughness causes the interface magnetization to be zero [14]. These results are very unusual since they are opposite to the conclusions which could be made by looking at a perfect $\mathrm{FeF}_{2}$ spin lattice. In that approach, for single domain $\mathrm{FeF}_{2}$ crystals with perfectly smooth surfaces [(110), (100), and (001)] (see Fig. 1 in Ref. [4], for example), the (100) and (001) surfaces are magnetically uncompensated, whereas the (110) surface is nominally compensated.

Furthermore, the $m^{\prime} m 2^{\prime}$ subgroup of the (110) surface contains no elements that interchange the two magnetic sublattices in $\mathrm{FeF}_{2}$. Therefore, the equivalence of the two sublattices is broken at the (110) interface; the resulting boundary magnetization is not a relativistic effect and is generally expected to be large [14]. Inequivalence of the magnetic sublattices at the (110) surface can be understood in simple terms by inspecting the magnetic structure of the crystal in a single-domain state with a surface that has atomic steps, as schematically shown in Fig. 5. The two magnetic sublattices are shown using green (sublattice 1) and orange (sublattice 2) arrows pointing in and out of the page. All Fe atoms in sublattice 1 are bonded with $\mathrm{F}$ atoms above and below, whereas those in sublattice 2 with $\mathrm{F}$ atoms in front and in the back. While these bonds are equivalent in the bulk, they are crystallographically distinct near the surface, and thus the near-surface $\mathrm{Fe}$ atoms in sublattices 1 and 2 are in different chemical environments [21]. Generally speaking, these atoms may have different magnetic moments [22]. Moreover, their chemical inequivalence is likely to result in a surface termination with a preferred exposure of one sublattice to the surface and to the $\mathrm{Cu}$ atoms in the neighboring layer, yielding a net magnetization at the surface. Thus, macroscopically the (110) surface of $\mathrm{FeF}_{2}$ has a net magnetization, which is responsible for the observed GMR.
Vanishing of the GMR measured in the 90-kOe magnetic field above the bulk $\mathrm{FeF}_{2}$ Néel temperature (78 K) (Fig. 2) confirms that the GMR signal is related to the antiferromagnetic order in $\mathrm{FeF}_{2}$. The uncompensated magnetization at the $\mathrm{FeF}_{2}(110) / \mathrm{Cu}$ interface arises from the same magnetic moments that constitute the antiferromagnetic spin lattice, and, hence it is linearly exchange coupled $[13,14]$ to the bulk $\mathrm{FeF}_{2}$ antiferromagnetic order parameter. This yields a strong pinning of the boundary magnetization along the direction of the bulk easy axis. The magnetic field couples to the boundary magnetization and thereby to the bulk antiferromagnetic order parameter. Therefore, field cooling creates a preference for one of the two antiferromagnetic domains in $\mathrm{FeF}_{2}$ and imposes the direction of the pinned magnetization. Once the AFSV is cooled below the Néel temperature, the boundary magnetization is "frozen in" and the external field can no longer reorient it. This explains why, at $10 \mathrm{~K}$, the pinned magnetization aligns parallel to the $\mathrm{FeF}_{2}$ easy axis regardless of the orientation of the cooling field. In order to permanently reverse this boundary magnetization after field cooling, an entire antiferromagnetic domain must be switched, whereas the Zeeman coupling is only present at the surface. Since $\mathrm{FeF}_{2}$ has a large uniaxial anisotropy, this reversal requires a very strong magnetic field at low temperatures. If the $\mathrm{FeF}_{2}$ layer is sufficiently thick, Zeeman coupling at the surface becomes ineffective, and switching may only occur through the bulk spin-flop transition. This explains why the GMR signal measured at $10 \mathrm{~K}$ remains unchanged as $H_{\text {meas }}$ is varied from 5 to $90 \mathrm{kOe}$ [Fig. 3(a)].

\section{Isothermal reversal of the pinned boundary magnetization}

The fact that the GMR measured at $10 \mathrm{~K}$ remains the same in the entire $H_{\text {meas }}$ range confirms the unprecedented stability of the pinned magnetization at low temperatures. At the same time, the suppression of the GMR signal by a strong magnetic field at 65 and $70 \mathrm{~K}$ [Fig. 3(a)] indicates that such fields affect the microscopic spin structure of $\mathrm{FeF}_{2}$ in the vicinity of the paramagnetic transition. In particular, this explains the abrupt drop near $60 \mathrm{~K}$ in the temperature dependence of GMR (Fig. 2), and vanishing signal above the critical field of $80 \mathrm{kOe}$ $(55 \mathrm{kOe})$ at $65 \mathrm{~K}(70 \mathrm{~K})$ in the field dependencies of GMR [Fig. 3(a)]. Two microscopic mechanisms of the boundary magnetization reversal can be envisioned. First, due to reduction of anisotropy, the boundary magnetization may tend to align parallel to the external magnetic field, thereby remaining parallel to the Co-layer magnetization, which would result in the absence of the GMR signal. Second, close to the Néel temperature of $\mathrm{FeF}_{2}$, the external field below $90 \mathrm{kOe}$ may induce either a spin-flop transition or a transition to a paramagnetic state [23-25]. As a result, either the boundary magnetization and the bulk antiferromagnetic order parameter would align perpendicular to the external field, or the antiferromagnetic order would disappear, respectively. In both cases, the boundary magnetization would rotate in unison with the external magnetic field. This, in turn, would yield the absence of the GMR signal.

According to Fig. 3(a), if the AFSV is oriented at $\theta_{\text {cool }}$ while $H_{\text {meas }}$ is changed, then pinning is completely restored in low magnetic fields. Thus, the ramp-up and ramp-down 
branches almost coincide for $65-\mathrm{K}$ and $70-\mathrm{K}$ curves in Fig. 3(a). This means that pinning can be erased and restored isothermally, slightly below the Néel temperature, without repeating the cooling procedure. Moreover, the angular dependence of the resistance is almost unchanged after application of a 50-kOe magnetic field [Figs. 3(b) and 3(c)]. In contrast, the minimum of the resistance shifts by $180^{\circ}$ after subjecting the valve to magnetic field of $70 \mathrm{kOe}$ [Fig. 3(d)]. These observations can be interpreted as follows. Since $50 \mathrm{kOe}$ is below the GMR suppression field at $70 \mathrm{~K}$, applying this field in the direction opposite to the cooling field does not affect the pinned magnetization at the $\mathrm{FeF}_{2}$ interface. In contrast, the 70-kOe magnetic field applied opposite to the cooling field reverses the pinned boundary magnetization, which is subsequently pinned when the magnetic field is ramped down to 10 kOe [Fig. 3(d)]. Overall, these measurements demonstrate that, at temperatures slightly below the Néel temperature, the pinned magnetization at the boundary of $\mathrm{FeF}_{2}$ may be reversed by applying a sufficiently strong magnetic field. Such reversal of the boundary magnetization in a strong magnetic field at temperatures close to the Néel temperature may be employed for isothermal imprinting of pinning.

\section{E. Further considerations}

Since the pinned uncompensated magnetization can induce the exchange bias, many phenomena observed in $\mathrm{FeF}_{2} / \mathrm{Cu} / \mathrm{Co}$ spin valves are directly relevant to the phenomena observed in bilayers composed of epitaxial $\mathrm{FeF}_{2}$ and ferromagnetic layers. First, strong exchange bias has been observed in $\mathrm{FeF}_{2}(110) /$ ferromagnet bilayers, whereas the exchange bias in $\mathrm{FeF}_{2}(100) /$ ferromagnet and $\mathrm{FeF}_{2}(001) /$ ferromagnet bilayers is weak [4]. Second, in Ref. [26], it was shown that applying a high magnetic field at temperatures slightly below the Néel temperature strongly affects the exchange bias field in $\mathrm{MnF}_{2} / \mathrm{Fe}$ bilayers. Similarly to the $\mathrm{FeF}_{2}(110) / \mathrm{Cu} / \mathrm{Co}$ valve, the effect emerges due to the reversal of the antiferromagnetic spin structure in the vicinity of a paramagnetic phase [25]. Third, in $\mathrm{FeF}_{2}(110) / \mathrm{Co}$ bilayers, strong pining of the boundary magnetization along the $\mathrm{FeF}_{2}$ easy [001] axis was found to be dependent only on the projection of the cooling field on the easy axis, and not on the cooling field direction [20]. Finally, our observations suggest that, at temperatures close to the Néel temperature of $\mathrm{FeF}_{2}$, exchange bias may be imprinted isothermally in a $\mathrm{FeF}_{2}(110) /$ ferromagnet bilayer by applying magnetic field strong enough to overcome the anisotropy of $\mathrm{FeF}_{2}$.

A series of experiments demonstrated that defects inside the $\mathrm{FeF}_{2}$ layer and at its interface strongly influences the exchange bias, and hence magnetization pinning, in the $\mathrm{FeF}_{2} /$ ferromagnet bilayers [27-29]. Such defects may be pinned after field cooling and contribute to GMR. It is impossible to exclude the role of the defects on the GMR in the $\mathrm{FeF}_{2}(110) / \mathrm{Cu} / \mathrm{Co}$ AFSV, and that these defects might also be pinned after field cooling. For this case, it is hard to predict the relative strength their contribution to the observed GMR. Moreover, it might be difficult to separate it from the contribution by the mechanism proposed above. However, the symmetry mechanism proposed in this work is intrinsic and is not affected by defects. Moreover, the general properties of the boundary magnetization following from symmetry also apply to defects located near the surface. It is important that the symmetry mechanism yields a robust explanation for the dependence of uncompensated surface magnetization on crystalline orientation of the $\mathrm{FeF}_{2}$ layer.

We should point out that the AFSV technique was previously used to detect the strongly pinned uncompensated magnetization at the $\mathrm{FeMn}-\mathrm{Cu}$ interface [11]. There are two important differences between those devices and the AFSV studied here. First, the FeMn layer in Ref. [11] was polycrystalline, whereas the $\mathrm{FeF}_{2}$ layer is epitaxial, which allowed us to study $\mathrm{FeF}_{2}$-based valves with different crystallographic orientations. This analysis leads to a clear conclusion on the relationship between the crystallographic symmetry and magnetization of the interface. Second, the fact that $\mathrm{FeF}_{2}$ is an insulator allows us to connect directly the behavior of the magnetoresistance to the $\mathrm{FeF}_{2}-\mathrm{Cu}$ interfacial magnetization.

\section{CONCLUSION}

Sensitive magnetotransport measurements of AFSVs containing an epitaxial $\mathrm{FeF}_{2}$ layer confirm the presence of pinned uncompensated magnetization at the $\mathrm{FeF}_{2}(110) / \mathrm{Cu}$ interface, as expected from symmetry considerations. Due to strong coupling of this uncompensated magnetization to the staggered bulk $\mathrm{FeF}_{2}$ spin structure, which has high anisotropy, this magnetization is unusually stable below the Néel temperature (78 K). Specifically, a magnetic field as high as $90-\mathrm{kOe}$ has no effect on the pinning at $10 \mathrm{~K}$. Close to the Néel temperature, the anisotropy of $\mathrm{FeF}_{2}$ anisotropy is reduced, and the reversal of the interfacial magnetization becomes possible. This effect can be used for isothermal imprinting of the boundary magnetization.

$\mathrm{FeF}_{2}$ is the second insulating antiferromagnet, after $\mathrm{Cr}_{2} \mathrm{O}_{3}$ [16], in which the surface magnetization was confirmed experimentally. In contrast, the presence of time-reversal symmetry forbids roughness-insensitive boundary magnetization in $\mathrm{NiO}$ and $\mathrm{CoO}$. The AFSV technique developed here can also be used to probe the surface magnetization in metallic $\mathrm{CuMnAs}$ and $\mathrm{Mn}_{2} \mathrm{Au}$ antiferromagnets whose spin structures can be manipulated electrically [30,31]. The absence of a macroscopic time-reversal symmetry in those materials implies that their (001) surfaces must have a surface magnetization coupled to the bulk antiferromagnetic order parameter, similar to the $\mathrm{FeF}_{2}(110)$ surface considered here. We emphasize that the GMR technique can be used to detect $180^{\circ}$ switching of the antiferromagnetic order parameter. This aspect may make this detection method attractive for applications in antiferromagnetic memory devices.

\section{ACKNOWLEDGMENTS}

This work was supported by the Office of Basic Energy Science, US Department of Energy, BES-DMS funded by the Department of Energy's Office of Basic Energy Science, DMR under Grant No. DE FG02 87ER-45332. K.B. is supported by the NSF through Grants No. DMR-1609776, No. DMR-1916275, and Nebraska Materials Research Science and Engineering Center (MRSEC) Grant No. DMR-1420645. 
[1] V. Baltz, A. Manchon, M. Tsoi, T. Moriyama, T. Ono, and Y. Tserkovnyak, Rev. Mod. Phys. 90, 015005 (2018).

[2] K. Olejník, T. Seifert, Z. Kašpar, V. Novák, P. Wadley, R. P. Campion, M. Baumgartner, P. Gambardella, P. Němec, J. Wunderlich, J. Sinova, P. Kužel, M. Müller, T. Kampfrath, and T. Jungwirth, Sci. Adv. 4, eaar3566 (2018).

[3] R. Cheng, D. Xiao, and A. Brataas, Phys. Rev. Lett. 116, 207603 (2016).

[4] J. Nogués, T. J. Moran, D. Lederman, I. K. Schuller, and K. V. Rao, Phys. Rev. B 59, 6984 (1999).

[5] C. Tsang, N. Heiman, and K. Lee, J. Appl. Phys. 52, 2471 (1981).

[6] M. J. Carey and A. E. Berkowitz, Appl. Phys. Lett. 60, 3060 (1992).

[7] R. Jungblut, R. Coehoorn, M. T. Johnson, Ch. Sauer, P. J. van der Zaag, A. R. Ball, Th. G. S. M. Rijks, J. aan de Stegge, and A. Reinders, J. Magn. Magn. Mater. 148, 300 (1995).

[8] P. J. van der Zaag, A. R. Ball, L. F. Feiner, R. M. Wolf, and P. A. A. van der Heijden, J. Appl. Phys. 79, 5103 (1996).

[9] H. Ohldag, A. Scholl, F. Nolting, E. Arenholz, S. Maat, A. T. Young, M. Carey, and J. Stöhr, Phys. Rev. Lett. 91, 017203 (2003).

[10] J. Nogués and I. K. Schuller, J. Magn. Magn. Mater. 192, 203 (1999).

[11] P. N. Lapa, I. V. Roshchin, J. Ding, J. E. Pearson, V. Novosad, J. S. Jiang, and A. Hoffmann, Phys. Rev. B 95, 020409(R) (2017).

[12] B. G. Park, J. Wunderlich, X. Martí, V. Holý, Y. Kurosaki, M. Yamada, H. Yamamoto, A. Nishide, J. Hayakawa, H. Takahashi, A. B. Shick, and T. Jungwirth, Nat. Mater. 10, 347 (2011).

[13] A. F. Andreev, JETP Lett. 63, 758 (1996).

[14] K. D. Belashchenko, Phys. Rev. Lett. 105, 147204 (2010).

[15] L. D. Landau, L. P. Pitaevskii, and E. M. Lifshits, Electrodynamics of Continuous Media (Butterworth-Heinemann, Oxford, 1984), Sec. 51.

[16] X. He, Y. Wang, N. Wu, A. N. Caruso, E. Vescovo, K. D. Belashchenko, P. A. Dowben, and C. Binek, Nat. Mater. 9, 579 (2010).
[17] C. Hahn, G. de Loubens, V. V. Naletov, J. Ben Youssef, O. Klein, and M. Viret, Europhys. Lett. 108, 57005 (2014).

[18] S. M. Rezende, R. L. Rodríguez-Suárez, and A. Azevedo, Phys. Rev. B 93, 054412 (2016).

[19] J. Olamit, E. Arenholz, Z.-P. Li, O. Petracic, I. V. Roshchin, R. Morales, X. Batlle, I. K. Schuller, and K. Liu, Phys. Rev. B 72, 012408 (2005).

[20] J. Olamit, Z.-P. Li, I. K. Schuller, and K. Liu, Phys. Rev. B 73, 024413 (2006).

[21] S. López-Moreno, A. H. Romero, J. Mejía-López, A. Muñoz, and I. V. Roshchin, Phys. Rev. B 85, 134110 (2012).

[22] D. Lederman, R. Ramírez, and M. Kiwi, Phys. Rev. B 70, 184422 (2004).

[23] S. M. Rezende, J. Phys. C 11, L701 (1978).

[24] V. Jaccarino, A. R. King, M. Motokawa, T. Sakakibara, and M. Date, J. Magn. Magn. Mater. 31-34, 1117 (1983).

[25] Y. Shapira and S. Foner, Phys. Rev. B 1, 3083 (1970).

[26] J. Nogués, L. Morellon, C. Leighton, M. R. Ibarra, and I. K. Schuller, Phys. Rev. B 61, R6455 (2000).

[27] A. C. Basaran, T. Saerbeck, J. de la Venta, H. Huckfeldt, A. Ehresmann, and I. K. Schuller, Appl. Phys. Lett. 105, 072403 (2014).

[28] J. Nogués, D. Lederman, T. J. Moran, I. K. Schuller, and K. V. Rao, Appl. Phys. Lett. 68, 3186 (1996).

[29] F. Torres, R. Morales, I. K. Schuller, and M. Kiwi, Nanoscale 9 , 17074 (2017).

[30] P. Wadley, B. Howells, J. Železný, C. Andrews, V. Hills, R. P. Campion, V. Novák, K. Olejník, F. Maccherozzi, S. S. Dhesi, S. Y. Martin, T. Wagner, J. Wunderlich, F. Freimuth, Y. Mokrousov, J. Kuneš, J. S. Chauhan, M. J. Grzybowski, A. W. Rushforth, K. W. Edmonds et al. Science 351, 587 (2016).

[31] I. A. Zhuravlev, A. Adhikari, and K. D. Belashchenko, Appl. Phys. Lett. 113, 162404 (2018).

Correction: A grant number was missing in the Acknowledgment section and has been inserted. 